\title{
RADIO FREQUENCY IDENTIFICATION IN CYBER-PHYSICAL SYSTEMS
}

\author{
Kamila Mašková, Eng., Director IFM GmBH in Czech Republic, Slovakia and Eastern Europe, \\ Iryna Petrovska, Ph.D., As.-Prof.; Oleksander Ryshkovskyy, Ph.D., As.-Prof.; \\ Svyatoslav Yatsyshyn, Dr. Sc., Prof.; e-mail: rysch@gmx.at \\ Lviv Polytechnic National University, Ukraine
}

\begin{abstract}
Automated systems that are part of cyber-physical systems provide distribution of goods or prefabricated components, as well as their documenting and automatic tracking in real-time. Workpieces are marked with RFID transponders. RFID antennas allow contactless reading and/or recording of information, RFID electronics process and transmit it to the programmable logic controller via PROFIBUS DP, PROFINET, Ethernet IP, Ethernet TCP/IP, or EtherCAT interface. The peculiarities of the universal interface for the needs of cyber-physical systems equipped with RFID means and the main metrological characteristics of the latter are studied in the work.

Key words: Automated identification systems, ID-transponder, Identification parameters, IO-Link software.
\end{abstract}

\section{Introduction}

Radio Frequency IDentification (further - RFID) is a method of automatic identification of objects, in which the data stored in the so-called RFID tags (transponders) are read or written utilizing radio signals [1-4]. The task of the RFID system consists of ensuring the storage of the received information stored in digital form, and then in its transmission to perform identification processes. Radiofrequency identification is used for such purposes as access control, vehicle identification, inventory control, production automation, movement of cargo and transport flows, automation of warehousing, automation of loading and unloading. RFID is based is a contactless technology that requires neither contact with the reader nor direct visibility of the object being read (as in barcode technology). That's why RFID removes the problems associated with "contact" technology. High-quality reading is guaranteed in heat, rain, cold, contamination with fat, or other substances.

For example, transponders are used in vehicle keys and are the transceivers that allow the owner to be identified by a car immobilizer unit. The automotive industry uses two types of transponders: cloned and programmable. Cloned transponders do not contain factoryprogrammed data and are copied applying special equipment. Programmable transponders contain data previously individually programmed at the factory, this data is also recorded in the car immobilizer unit by diagnostic and special equipment. When the ignition is switched on, the car antenna generates an electromagnetic field. The latter feeds the transponder chip located in the ignition key. The transponder uses the power of the electromagnetic field to transmit a unique code stored in its memory. If the transmitted code corresponds to the data stored in the immobilizer, the car starts.

\section{Drawbacks}

There exist problems with radio frequency object identifying. First, it is embedded in the mentioned means of metrological characteristics, which are manifested by the parameters of identification. Insufficient identification of characteristics leads to a scatter of identification parameters. The latter include the identification sensitivity associated with the threshold distance and many other characteristics. Second, the metrological support of RFID means of objects, which includes according to [5] methods and means of support, is only guaranteed by the manufacturer.

\section{The Goal of the Paper}

The purpose of the current paper is to study and metrological substantiation of the software and hardware of radio frequency identification for the cyber-physical system needs.

\section{Metrological Approach to the Study} of RFID Sensors' Performance

4.1. Research of transponder characteristics for aviation

The most responsible conditions for the transponders' application seem to be ensuring the aircraft landing at airports. After all, the speed of the aircraft during landing is about $100 \mathrm{~m} / \mathrm{s}$. Then a slight error in the displacement from the centerline of the runway can lead to the landing of the aircraft outside its limits.

A common type of error occurs when a transponder transmits incorrect position data. On some aircraft, such as the older Airbus A300, Boeing 737, 757, or 767, position data is embedded in the microprocessor on the ground before takeoff and may change during the flight. This error is complicated within the flight, altering with direction and strength of winds and circular schemes of approach, etc. Another common type of error and even transponder errors are due to the following. To identify and match the existing databases of a particular tool where the transponder is installed, use the unique code of the latter. If the code is incorrect, the wrong 
flight is mistakenly set for identification. We do not consider this kind of error in the future. If there is a problem with the signal intensity, for example, during takeoff, landing, or low altitude flight, a small synchronization error can lead to significant errors in locating the object with the transponder [6].

A separate study of the characteristics of transponders was carried out by [7]. There were some errors related to the movement of aircraft and its technical equipment, including transponders. They are mainly due to the relatively high rate of the aircraft's position change during landing. Besides, errors associated with the establishment of the spatial coordinates at the particular time before landing, include the height error, the error caused by offset from the centerline of the runway, etc. Therefore, strict requirements are set for the parameters of the transponder pulses that are sent. Namely, are normalized: the position of a particular pulse relative to all other pulses with an offset error of fewer than $100 \mathrm{~ns}$.; the average interval between the first and fourteenth possible position of the pulse (20.2-20.4 $\mu \mathrm{s}$ ); the pulse width of the response (for example, the average pulse width is from 0.35 to $0.55 \mu \mathrm{s}$ ); response delay - about $0.2 \ldots 3.5 \mu \mathrm{s}$, depending on the mode.

\subsection{RFID sensors for Industry 4.0}

The specificity of the operation of these sensors in Industry 4.0 [1] is determined by areas of modern technology. At significant distances of writing and reading, data exchange depends on the RFID antenna (Fig. 1). Production data and quality parameters can be stored in the identification label using an antenna type.

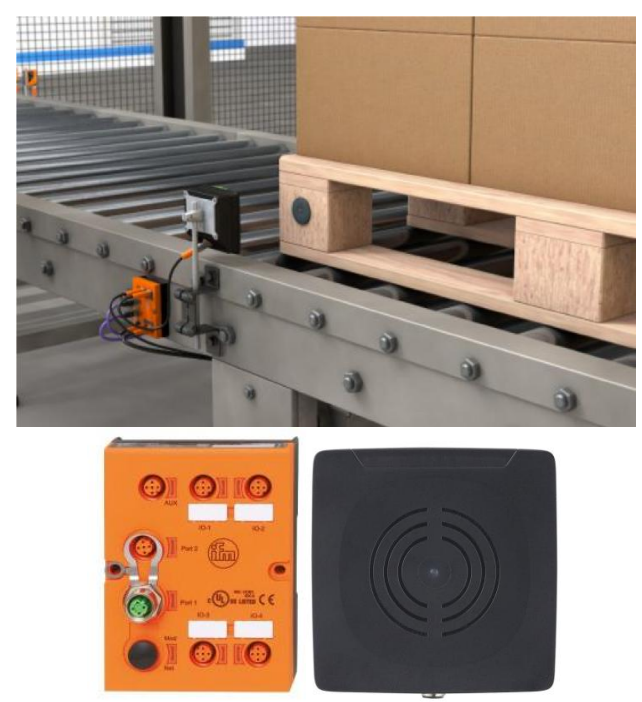

Fig. 1. Data exchange via RFID means. Communication devices are shown in magnification on the left and right

Automotive industry. The coordinated processing centers, transport systems, and industrial robots form the preconditions for the realization of the serial production, and at the same time meet the specific equipment requirements. Every single process in the automotive industry - press, body, or paint shop, power transmission, and final assembly - must work reliably and with a high level of availability of equipment and installations. A) Each seat has to be identified by a built-in RFID tag and can thus be attached to the appropriate vehicle, as shown in Figure 2.

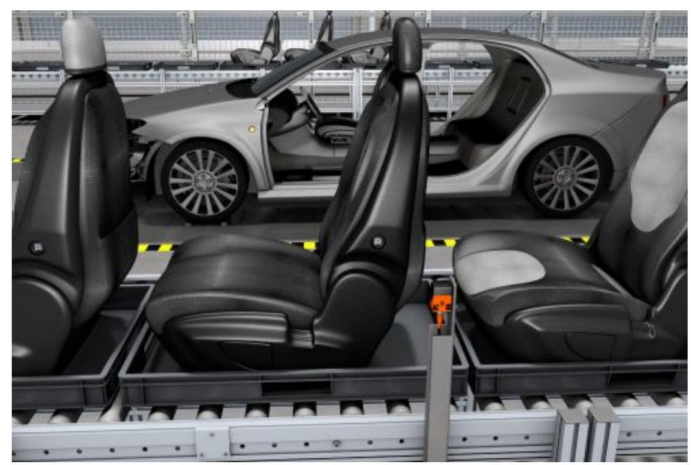

Fig. 2. Identification of seat kits using RFID.

The RFID system is a flexible system for manufacturing and handling technologies. It is available for all types of industrial systems. The antenna is connected without complicated wiring. IFM Electronics LINERECORDER modules are used for product tracking, order processing, and component management. Figure 3 shows a set for identifying the seats [3].

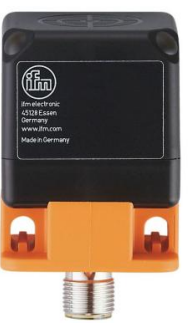

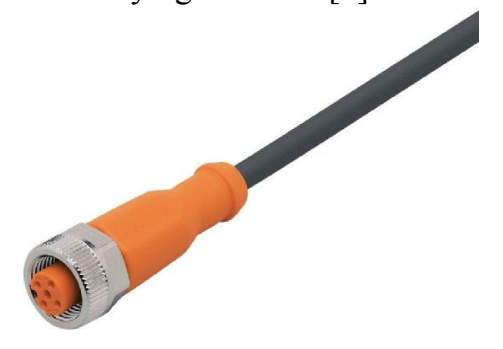

$b$
Fig.3. Set for identification of the seats: a) ANT513 RFID HF read/write head; b) EVCO02 connecting cable

B) Painted car bodies are moved on skids in a multilevel warehouse. At any time, the RFID system can determine the location of the runway in any area. C) Identification of containers for details in the transmission assembly shop is also carried out using RFID. In automated manufacturing, the latter is used, for example, to detect details/containers. The DTA3000 read/write head and the E80311 identification tag determine the current location at work. With LINERECORDER SENSOR (ZGS210) you can use the modules of "tracking" and order processing. This means that at any time it is known at what level of production is which product. Products for this application include means for identifying containers: a) DTA300 read/write head with AS-interface; b) E80311 RFID tag [3]. 
Tracking of sheet metal components using RFID. The RFID-UHF system is optimized the production control (flow of materials and components, in the field of tracking and supply chain management). Component information is read and codes are stored. This provides complete tracking of consignments. The kit for tracking sheet metal components includes: a) E80351 RFID tag; b) DTE801 compact RFID device; c) E80331 connecting cable.

Food and meat processing industry. Process reliability and quality control with the ANT513 RFID HF read/write head are necessary for the production of beverages, confectionery, and dairy as well as in meat processing. Here, the slightest pollution leads to the recall of products from the market or downtime of production lines.

Photoelectric detection of packing boxes in conveyor systems in cutting lines. High-precision, fully automatic tracking of boxes is carried out utilizing RIFD systems. RFID DTE100 electronics with Profibus-DP interface is optimized for quality assurance. In combination with antennas and transponders, RFID systems can be used in the production process as an electronic route map. Due to the degree of protection IP 67 , it is also applied in conveyor technology and heavy operating conditions. The ANT411 RFID HF read/write head and the industrially compatible, rugged antennas in the M12 enclosure can be used in confined spaces, as is often with automation and manipulation technologies. They are also suitable for the food industry if they are not in direct contact with the environment.

Monitoring in industries where disinfection is carried out regularly. Here, feedback is provided on the position of the distribution panel's ell used for manual transition between different lines (Fig. 4). IGT241 sensors made of stainless steel can be connected, for example, to Profibus or Ethernet with help of the appropriate DTE evaluation electronics [3] and are characterized by detergents' resistance (Ecolab certificate). Their commu- nication is provided by compact RFID M12 antennas. Ells of the pipe position feedback kits on the distribution panel; a) IGT260 inductive sensor in all-metal housing; b) DTI424 RFID HF read/write head; c) AL1303 IOLink master with Profinet interface; d) EVF515 connecting cable; e) E80371 RFID tag.

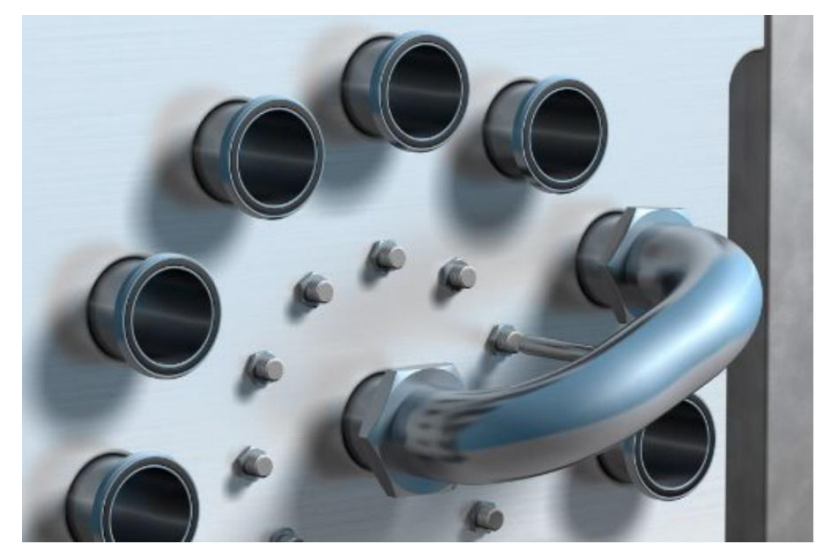

Fig. 4. Ell of the pipe on the distribution panel

Packaging industry. In addition to primary and secondary packaging, there are differences between solid and liquid products. There are developed different types of sensors, for example, to determine the position, volume, and level. RFID systems for product identification consist of the DTE101 evaluation electronics (type RFID LF/HF) and ANT600 RFID HF read/write head, help to update the range of products in this area.

Machine tool construction is divided into two main areas: processing of metals by pressure and by cutting. A) Identification and detection of interchangeable instruments is realized as follows. The tools are removed from the cassette using RFID detection (Fig.5). Photoelectric distance sensors such as O5D100 detect whether the selected instruments are in the mount. The ANT410 RFID HF read/write head and EVC003 connecting cable are used to receive and transmit information.

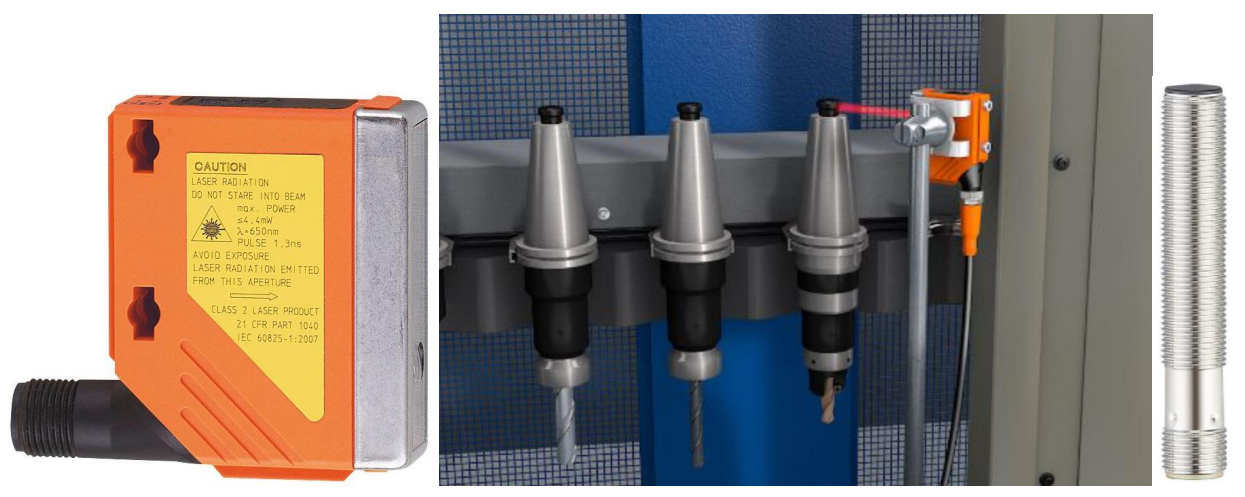

Fig.5. Identification of interchangeable instruments: at the left side

$s$ the photoelectric distance sensor type O5D100; at the right side is the read/write head type ANT410 RFID HF 


\section{Software and Metrological Support of Radio Frequency Identification Means}

When choosing sensors and RFID objects of developed systems, the distances between the recording and reading heads (depending on the tasks) should be taken into account, as well as, and most importantly, the possibility of software changing the sensors' characteristics [8-9]. Note that RFID sensors and systems from IFM Electronic operate based on the IO-Link communication interface.

\subsection{The main characteristics of the IO-Link} interface

A standardized IEC 61131-9 interface concerns for AS and gateways with automatic scanning of sensors in the network, including encoders, capacitive, photoelectric sensors, temperature, flow, pressure, and level sensors, as well as for basic field logic controllers and Fieldbus. IO-Link is a universal data interface, and IO-Link wizards are universal I/O modules. Different types of devices can work on one port: sensors, actuators, and devices with a switching signal. Thus, one IO-Link port can replace several components: analog (4 ... $20 \mathrm{~mA}, 0 \ldots 10 \mathrm{~V}$ ), binary input, and output cards.

Also, IO-Link is a smart interface for sensors, i.e. it can be integrated into smart sensors. Data exchange with the controller can take place in two directions: messages about diagnostics or maintenance, or data for remote adjustment of parameters. IO-Link provides easy setup when connecting and replacing sensors; while sensor parameters are stored in the wizard and transferred to a new sensor. There is an offline setting of the sensor parameters before its installation, as well as identification of the sensor. The latter is automatically recognized when using the IO-Link program on the Fieldbus. If the sensor is mishandled during operation, it can be switched off with the key. The received readouts are transmitted without data loss during conversion; the values on the sensor display correspond to the values on the equipment display. You can diagnose the information-measuring system to the level of the sensor without interrupting its operation. At the same time, the use of a three-wire cable reduces the number of terminals and frees up space in the cable channels.

IO-Link software and hardware provide opportunities for communication between the equipment control system and the field level: sensors and actuators become active participants in a comprehensive automation network. They independently report errors and conditions to the control device. As receivers, they obtain and process signals. The IO-Link control equipment, as a component of the I/O module, is installed in the switch cabinet or on the periphery as a remote $\mathrm{I} / \mathrm{O}$ module with IP65/67 protection. IO-Link is connected using a standard cable for sensors and actuators with a length of at least $20 \mathrm{~m}$ (Fig. 6).

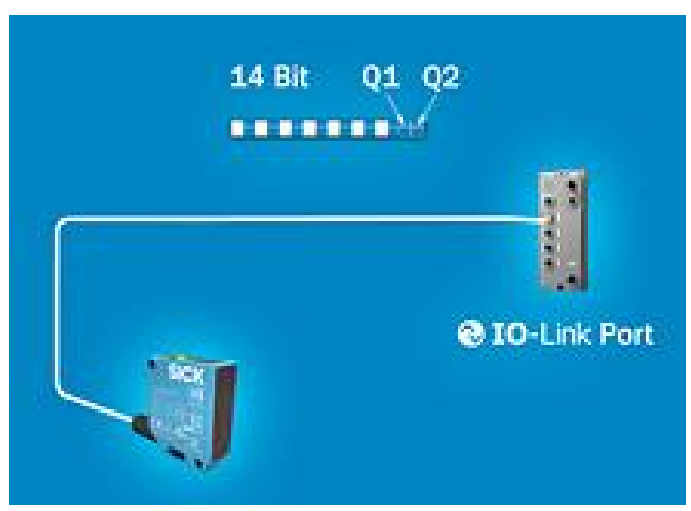

Fig. 6. Scheme of cable wiring without shielding

Operate with signals (binary switching, analog input/output), which are transmitted to IO-Link directly in digital form (Fig. 7).

IO-Link provides the following advantages in the implementation of standard functions: Flexible adjustment of the sensor; Prevention of erroneous settings due to automatic parameterization; Condition monitoring/ diagnostics; Minimize the risk of failure; Optimize the maintenance intervals and scheduled operation; Detection and elimination of critical states of sensors by a service engineer before failure; Sensor visualization and parameterization; Sensor diagnostics; Parameterization of advanced functions using visualization software; Checking and optimizing the quality indicators or functional reserves of the sensor signal; Simplified selection of sensor functions and performance according to production tasks; Easy adjustment of the sensor settings "on the desktop"; Digital transmission of analog-measured values; Parallel transmission of measured values and digital signals, etc.

Integration of the IO-Link sensor into the general automation system is carried out through the leading IOLink equipment (Fig. 8), and standard functional blocks (Fig. 9) simplify programming.

IO-Link control equipment includes IO-Link Ethercat main equipment, IO-Link Ethernet/IP master equipment, IO-LinkPROFINET. The corresponding control equipment is a subscriber of the industrial network or part of a modular input/output system connected to the industrial network. The latter's subscribers are integrated into the system through equipment (GSDML, EDS, ESI, etc.). When designing or configuring an automated structure, the necessary subscribers of the industrial network are determined and communication parameters are set. Programming becomes possible with the special functional unit of the IO-Link equipment, which simplifies acyclic communication between controllers and equipment, service data transmission, and process data interpretation. 


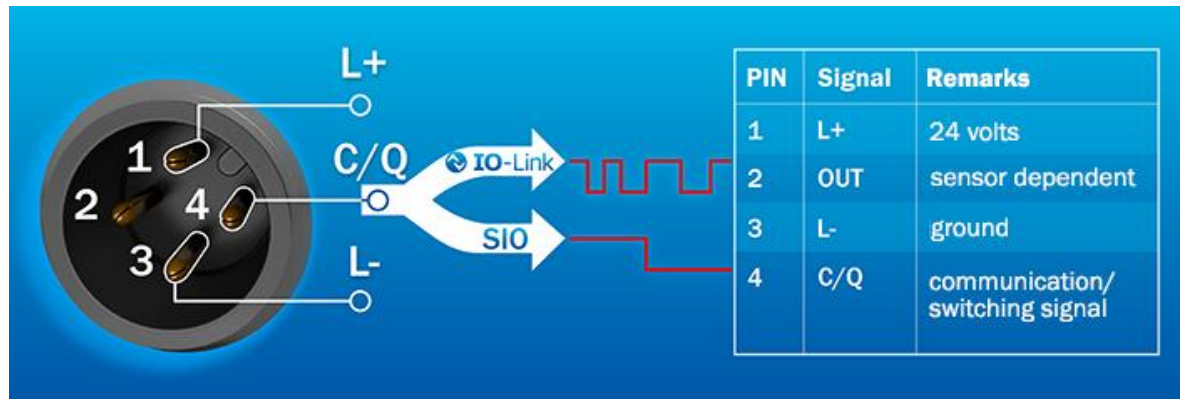

Fig. 7. IO-Link cable connection diagram

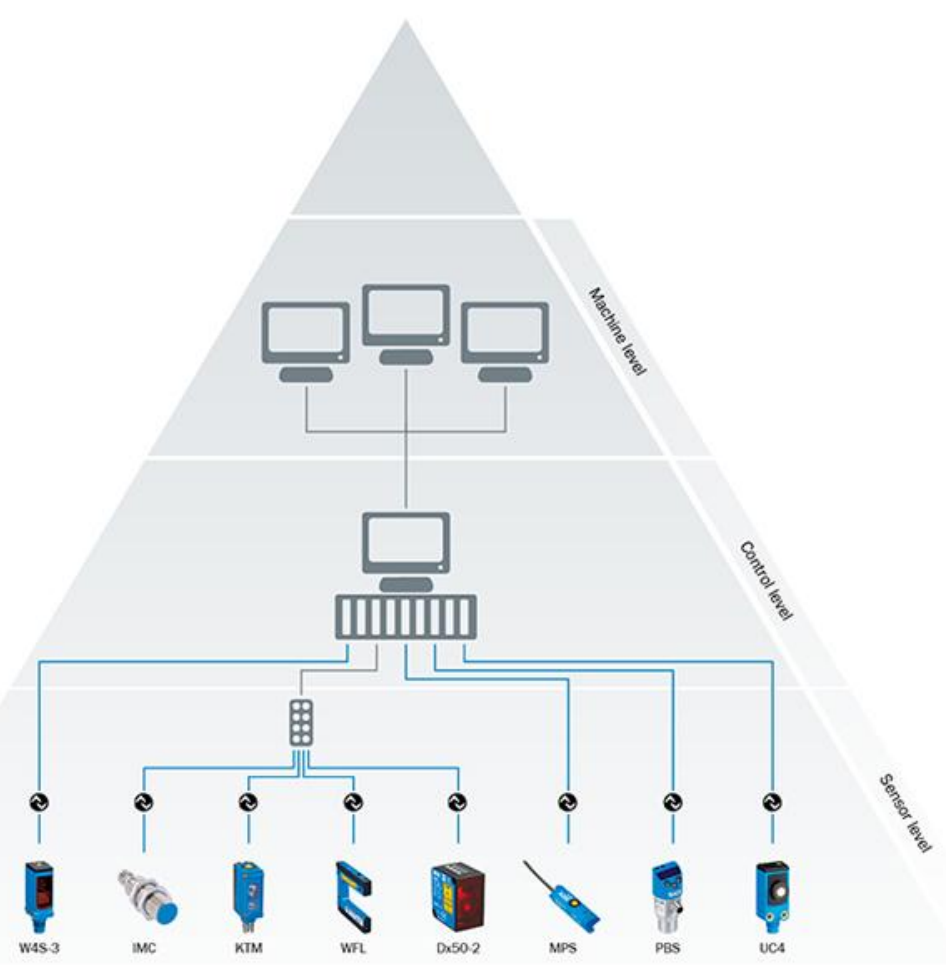

Fig. 8. Integration of the IO-Link sensor into the automation system
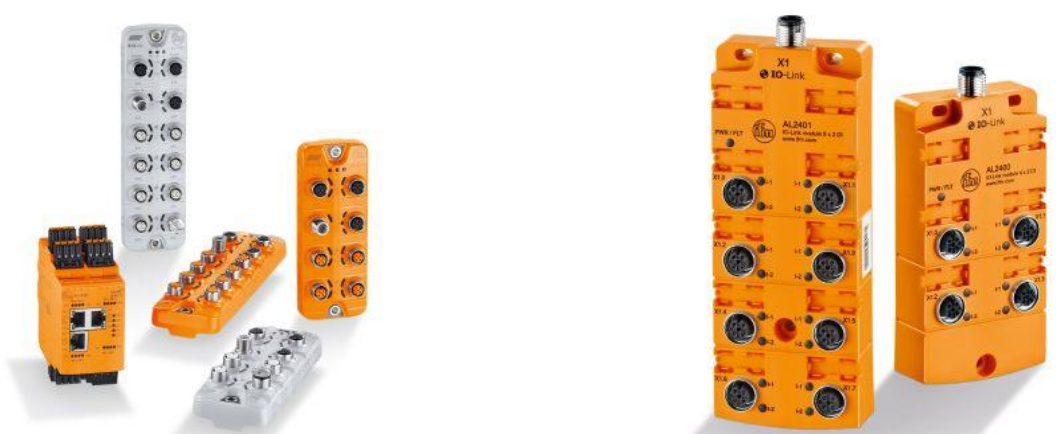

Fig. 9. IO-Link wizard and IO-Link modules 
The architecture of the network built based on this protocol consists of the IO-Link wizard, the IO-Link equipment - generally of sensors and drives, the standard three-wire connection scheme. The protocol supports 3-speed modes: $4.8 \mathrm{kbps}, 38.4 \mathrm{kbps}$, and 230 kbps. The system architecture looks like this (Fig. 10).

The network wizard has one or more ports. In this case, only one "managed" port can be connected to one port at a time, so IO-Link is a P2P interface, not a Fieldbus. After connecting the power, the equipment switches to $\mathrm{I} / \mathrm{O}$ mode. The behavior of the wizard depends on the configuration of its ports. If the port is in SIO mode, it behaves like a normal digital input. If the port configuration is set to communication mode, the wizard starts searching for new equipment. This process is called "waking up" (Fig. 11).

Communication between the control and the controlled devices is carried out according to the "requestresponse" scheme. First, the master initiates the transfer at maximum speed. If the connection attempt fails, the request is repeated at a slower speed. At the same time, specific equipment always supports only one speed. As soon as the master receives the answer, the data exchange begins. If the equipment is disconnected during the exchange, the wizard detects a disconnection and tries to restore it. If the attempt to restore the connection was successful (successful wake-up), the connection parameters have to be checked again. It may be necessary to validate them. Then the data exchange is resumed.

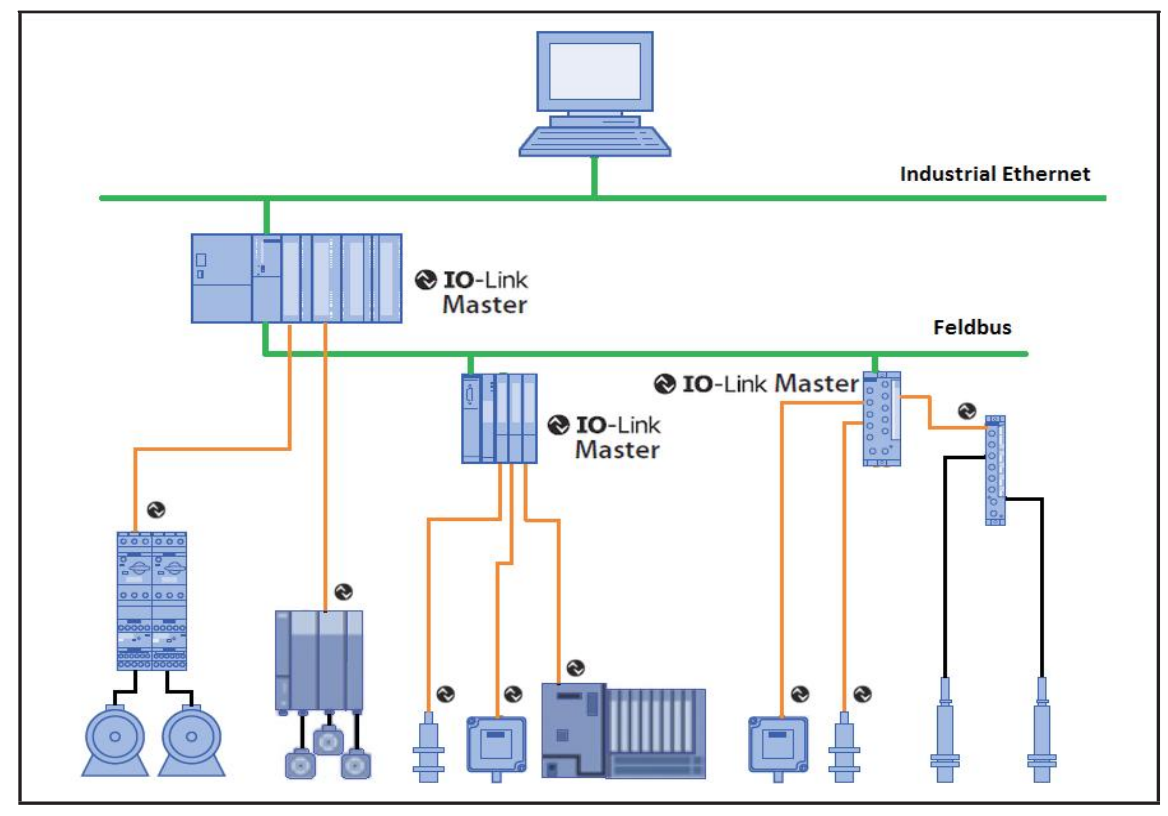

Fig. 10. IO-Link based network architecture

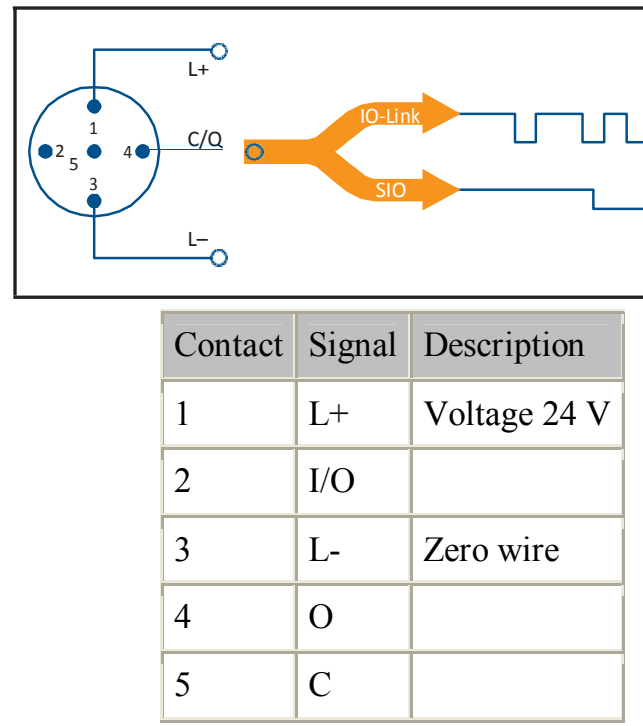

Fig. 11. Communication between control and managed devices 


\subsection{Optimization of metrological} characteristics of RFID sensors checking and assuring

When choosing RFID sensors and systems for the identification of objects, you must first take into account the distance between the recording and reading heads as well as the possibility of IO-Link software application. It should be noted that just RFID sensors and systems from IFM Electronic Work operate effectively with the IOLink communication interface.

\section{Conclusions}

To identify the objects in the construction of particular cyber-physical systems with radio frequency application, it is necessary studying such metrological characteristics as sensitivity and other parameters of each mean and conditions of their operation. This can be carried out by the corresponding software, for example, IO-Link for means of IFM Electronic. With help of the hardware-software metrological support, you can purposefully change the parameters of smart sensors and actuators to the requirements of a particular technology and, thus, implement flexible cyber-physical systems.

\section{Gratitude}

The authors are grateful to the Team of the Department of Information-Measuring Technologies of Lviv Polytechnic National University for an informative discussion on the obtained results.

\section{Conflict of interests}

The authors declare that there is no financial or other possible conflict related to the current paper.

\section{References}

1. Finkenzeller Klaus. (RFID Handbuch) - Fundamentals and Applications in Contactless Smart Cards, Radio Frequency Identification and Near-Field Communication, 2010, John Wiley \& Sons, Ltd. p. 480

2. Review on Security of Radio Frequency Identification Technology. Aastha, Preeti Gulia. Advances in Computational Sciences and Technology. Volume 10, Nu.8, pp. 2427-2433, 2017.

3. Markus Hansen, Sebastian Meissner: Identification and Tracking of Individuals and Social Networks using the Electronic Product Code on RFID Tags, IFIP Summer School, Karlstad, 2007.

4. Roy Want.An Introduction to RFID Technology. IEEE Pervasive Computing. January-March 2006, pp. 25-33, vol. 5.

5. M. Mykyichuk, Z. Kolodiy, S. Lazarenko, I. Likhnovskiy, Studies of Metrological Characteristics of Measuring Instruments, in Cyber-Physical Systems and Metrology 4.0. Editors S. Yatsyshyn, B. Stadnyk, IFSA Publishing, Barcelona, Spain, 2021.

6. Ian Petchenik's, https://www.flightradar24.com/ blog/common-errors-on-flightradar24/].

7. Nicholas J. Talotta. A Field Study of Transponder Performance in General Aviation Aircraft. FINAL REPORT DOT/FAA/CT-97/, Dec.5, 1997.

8. Álvarez López, Jacqueline Franssen, Guillermo Álvarez Narciandi, Janet Pagnozzi, Ignacio González-Pinto Arrillaga, Fernando Las-Heras Andrés. RFID Technology for Management and Tracking:e-Health Applications. Sensors 2018, 18, 2663 (www.mdpi.com/journal/sensors)

9. K. Ahsan, H. Shah, P. Ksngston. RFID Applications: An Introductory and Exploratory Study. JCSI Int. Journ. Comp. Sc. Iss., Vol.7, Issue 1, No.3, Jan. 2010. 